\title{
A DOR E DESCONFORTO NA PRÁTICA DO ALEITAMENTO MATERNO*
}

\author{
Alcimara Benedett ${ }^{1}$, Isilia Aparecida Silva ${ }^{2}$, Lucimare Ferraz $^{3}$, Patrícia de Oliveira ${ }^{4}$, \\ Elide Fragoso ${ }^{5}$, Joana Ourique
}

\begin{abstract}
RESUMO: A amamentação é uma prática de promoção à saúde e o conhecimento de fatores associados à interrupção é fundamental para aumentar o período da prática. Este estudo observacional, descritivo, transversal teve por objetivo conhecer os fatores de dor e de desconforto na prática do aleitamento materno. A população foi composta de mães de recém-nascidos, de dois a quatro meses, entre fevereiro e julho de 2012. Foram aplicados questionários em visitas

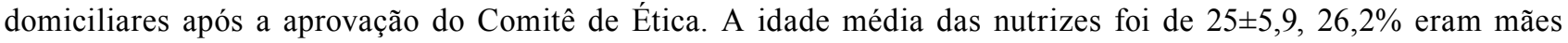
adolescentes. A posição preferida referida para amamentar é a sentada e o local de dor e de desconforto foi a mama. $\mathrm{O}$ sexo da criança teve significância estatística $(\mathrm{p}=0,038)$ em relação à dor e ao desconforto materno. Ações durante o pré-natal, sobre a técnica correta para evitar dor e desconforto, devem ser incentivadas evitando o desmame precoce. DESCRITORES: Aleitamento materno; Dor; Desmame.
\end{abstract}

\section{EL DOLOR Y MOLESTIA EN LA PRÁCTICA DEL AMAMANTAMIENTO MATERNO}

RESUMEN: El amamantamiento es una práctica de promoción a la salud y el conocimiento de factores asociados a la interrupción es fundamental para aumentar la duración de esta práctica. Este estudio observacional, descriptivo, transversal tuvo por objetivo conocer los factores de dolor y molestia en la práctica del amamantamiento materno. La población fue compuesta de madres de recién nacidos, de dos a cuatro meses, entre febrero y julio de 2012. Fueron aplicados cuestionarios en visitas domiciliares después de la autorización del Comité de Ética. La edad media de las nutrices fue de 25 $\pm 5,9 ; 26,2 \%$ eran madres adolescentes. La posición preferida para amamantar es la sentada y el local de dolor y molestia fue la mama. El sexo del niño tuvo significancia estadística $(\mathrm{p}=0,038)$ acerca del dolor y de la molestia materna. Acciones durante el prenatal, sobre la técnica correcta para evitar dolor y molestias, deben ser incentivadas evitando el desmame precoz.

DESCRIPTORES: Amamantamiento materno; Dolor; Destete.

\section{PAIN AND DISCOMFORT IN THE PRACTICE OF BREASTFEEDING}

ABSTRACT: Breastfeeding is a practice which promotes health, and knowledge of the factors associated with its interruption is fundamental for increasing the period of its practice. This observational, descriptive and cross-sectional study aimed to investigate the factors of pain and discomfort in the practice of breastfeeding. The population was made up of mothers of newborns aged between two and four months, between February and July 2012. Questionnaires were administered during a home visit following the approval of the Ethic Committee. The breastfeeding mothers' mean age was $25 \pm 5.9$, and $26.2 \%$ were adolescent mothers. The favourite position for breastfeeding mentioned was sat down, and the place most mentioned for pain and discomfort was the breast. The child's sex had statistical significance $(\mathrm{p}=0.038)$ in relation to the mother's pain and discomfort. So as to avoid early weaning, actions must be encouraged during the prenatal period regarding the correct technique for avoiding pain and discomfort. DESCRIPTORS: Breastfeeding; Pain; Weaning.

\footnotetext{
*Extraído do Trabalho de Conclusão do Curso de Medicina - Universidade Comunitária da Região de Chapecó, apresentado em 2012.

${ }^{1}$ Enfermeira. Doutora em Enfermagem. Professora da Universidade Comunitária da Região de Chapecó. Chapecó-SC-Brasil

${ }^{2}$ Enfermeira. Doutora em Obstetrícia. Professora da Universidade de São Paulo. São Paulo-SP-Brasil

${ }^{3}$ Enfermeira. Doutora em Saúde Coletiva. Professora da Universidade Comunitária da Região de Chapecó. Chapecó-SC-Brasil

${ }^{4}$ Médica. Doutora em Saúde da Criança e da Mulher. Professora da Universidade Comunitária da Região de Chapecó. Chapecó-SC-Brasil ${ }^{5}$ Médica.
} 


\section{INTRODUÇÃO}

$\mathrm{O}$ aleitamento materno (AM), além de suprir as necessidades nutricionais do lactente, oferece resistência contra infecções, estabelece vínculo psicológico entre a mãe e o recém-nascido, reduz má formação da dentição, estimula e exercita a musculatura que envolve o processo de fala, promove melhor a dicção e proporciona tranquilidade ao recém-nascido ${ }^{(1)}$. Além de atuar na defesa do organismo, o AM fortalece o vínculo mãe-bebê e é um alimento completo, constituído por vitaminas, minerais, gorduras, açúcares e proteínas $^{(2)}$.

As nutrizes permanecem por longo tempo e várias vezes ao dia em uma posição diferente das quais estavam habituadas antes de se tornarem mães. Por isso, as causas mais importantes para o desmame precoce entre nutrizes são dores ao amamentar e problemas na $\mathrm{mama}^{(3)}$. A postura, quando inadequada, torna-se incômoda, gera tensão muscular e interfere na lactação ${ }^{(4)}$. Contudo, as causas de dor e desconforto nas mães podem e devem ser prevenidas, ressaltando a importância das orientações no pré-natal e puerpério imediato, pelos profissionais da saúde, e da estimulação o mais precoce possível do aleitamento materno para ajudar as mães a praticarem a técnica de forma correta e indolor.

A Organização Mundial da Saúde, visando diminuir traumas mamilares e garantir a retirada eficiente do leite da mama pelo bebê, preconiza a promoção da técnica de amamentação ideal ${ }^{(5)}$. Assim, são necessárias ações para melhorar a técnica de amamentação, a fim de que as mães amamentem com mais conforto, aumentando as chances de ser mais prolongada e prazerosa $^{(6)}$.

O conhecimento dos fatores associados à interrupção da amamentação é fundamental para que a mulher tenha melhores condições para exercer esta prática. Assim, considerando que a dor e o desconforto podem ser elementos de desgaste para a amamentação, este estudo teve como objetivo conhecer os fatores de dor e desconforto, referido pelas nutrizes, na prática do aleitamento materno.

\section{MÉTODO}

Trata-se de um estudo observacional, descritivo, transversal, realizado com nutrizes atendidas em Unidades Básicas de Saúde (UBS) do Município de Chapecó, Santa Catarina. Foram selecionadas mulheres que estavam vinculadas às UBS de suas áreas residenciais. Para obter uma visão mais global e representativa do município, foram divididas as UBS de acordo com suas regiões norte, sul, leste e oeste. No total havia 130 puérperas assistidas nessas UBS e, destas, foram sorteadas, aleatoriamente, 42 mulheres, de acordo com o cálculo da amostra com erro probabilístico de $5 \%$. A partir dessa seleção, identificou-se o registro de vacinação das crianças, os endereços e contatos das puérperas cujos filhos tinham idade entre dois e quatro meses.

Foram incluídas apenas nutrizes primíparas, residentes do município de Chapecó, com filhos nascidos a termo.

O estudo consistiu na aplicação de três questionários, previamente estruturados, a saber: o primeiro questionário visava traçar o perfil socioeconômico da puérpera, contendo questões sobre as variáveis sociodemográficas; o segundo teve a finalidade de avaliar mamas e condições de amamentação ${ }^{(4)}$; e o terceiro avaliou o desconforto músculo-esquelético ligado à amamentação ${ }^{(4)}$, contendo questões sobre local, duração e intensidade da dor, e sua interferência ou não nas atividades de vida diária.

A análise estatística dos dados foi realizada com programa Statistical Package for Social Sciences (SPSS) versão 19.0 a partir de banco de dados digitado na forma Excel. O nível de significância adotado foi de $5 \%$, sendo os valores entre $5 \%$ e $10 \%$ considerados limítrofes.

A significância estatística entre as variáveis categóricas foi avaliada através de testes X2. Para as variáveis contínuas foram calculadas as medidas de tendência central (média, mediana) e de dispersão (variância, amplitude).

Este estudo foi aprovado pelo Comitê de Ética em Pesquisa da Unochapecó, sob o número 130/11. As participantes da pesquisa assinaram o Termo de Consentimento Livre e Esclarecido, após amplo esclarecimento.

\section{RESULTADOS}

A idade média das nutrizes entrevistadas foi $23 \pm 5,9$ anos, variando entre 15 e 39 anos. Identificaram-se $26,2 \%$ de mães adolescentes (16 a 19 anos de idade). Entre as entrevistadas, 95,2\% se declararam casadas. A renda per capita variou entre $\mathrm{R} \$ 150,00$ e $\mathrm{R} \$ 1.166,76$, sendo que $47,6 \%$ das mulheres tinham salário inferior a um salário mínimo. 
No que se referem às intercorrências do período de gestação, as mais relatadas foram pré-eclâmpsia $(9,5 \%)$ e ruptura precoce de membranas $(2,4 \%)$. Duas crianças $(4,8 \%)$ nasceram pequenas para a idade gestacional $(<2.500$ gramas). O parto do tipo cesárea foi o mais referido $(64,3 \%)$ entre as entrevistadas. E a maioria $(59,5 \%)$ referiu ter feito a primeira mamada na sala de recuperação pós-parto.

A média da frequência aproximada de mamadas do recém-nascido em 24 horas foi 11,2 2 ,9, variando entre 3 e 18 mamadas/dia. Observou-se que $35,7 \%$ das crianças têm preferência por um dos seios maternos para mamar. De todas as mães com mamilo plano, $25 \%$ referiram achar que o bebê não abocanha a mama com facilidade, enquanto apenas $8,8 \%$ das mães com mamilo normal (81\%) tinham esta queixa. Quanto à condição da mama, 40,5\% referiram ser flácidas e destas $35,3 \%$ afirmaram ter pequena quantidade de drenagem do leite.

Observou-se que a posição ao amamentar preferida pelas mães foi sentada $(95,2 \%)$, na qual o sofá foi o mobiliário da casa mais utilizado (76,2\%); $69 \%$ não utilizaram apoio para os pés e $11,9 \%$ usaram travesseiro na região lombar. Nenhuma das mães relatou ter uma postura ideal ao amamentar e $42,9 \%$ mencionaram que os ombros ficam tensos ao amamentar. A forma de apoiar a mama, em forma de tesoura, foi referida por $40,5 \%$ das mães.

Quanto à condição do lactente durante a amamentação, as nutrizes referiram que $11,9 \%$ dos lactentes eram sonolentos, $4,9 \%$ não abocanharem com facilidade a mama e $2,4 \%$ não tinham sugadas fortes, os demais $(80,8 \%)$ não apresentam dificuldades para mamar.

Quando questionadas se sentiam dor em alguma região do corpo, 35,7\% responderam sim. A região a qual mais se referiram foi a mama $(35,3 \%)$. Considerando as menções à dor na mama, 66,7\%, tiveram as fissuras como causa. Quanto à intensidade da dor, $66,7 \%$ referiram dor de moderada a forte; porém as mães declararam tentar superar esses sintomas através da mudança de posições para amamentar. Dor na região lombar foi a segunda queixa mais mencionada $(23,5 \%)$ pelas mães. Contudo, de acordo com elas, não atrapalha nas atividades diárias nem com o bebê. O restante dividiu-se igualmente no relato de dor em membro superior ou no pescoço. De todos os dados descritos, apenas o sexo do bebê foi estatisticamente significativo $(\mathrm{p}=0,038)$, sugerindo que a amamentação dos bebês do sexo masculino causam menos dor e desconforto durante a amamentação.

\section{DISCUSSÃO}

Como neste estudo foram entrevistadas apenas primíparas e a idade média das mães foi 23 anos, sugere-se que a falta de experiência em relação à maternidade pode contribuir para que a mãe tenha problemas durante a amamentação, podendo levar à dor e ao desconforto. Em um estudo de corte foi observado que quase metade das mães não tinham qualquer experiência anterior com a amamentação e que metade encontrou dificuldades para amamentar nos primeiros dias pós-parto ${ }^{(7)}$. Sugerindo, desse modo, que se a mãe não for orientada corretamente ela é uma potencial candidata a ter dor ou desconforto, em alguma parte do corpo, devido à técnica ao amamentar.

A baixa renda classicamente é associada a fatores que levam à interrupção precoce da amamentação devido às várias condições sugestivas: ter que trabalhar e não ter conhecimento de como continuar oferecendo seu leite; falta de conhecimento de como amamentar corretamente, prática incorreta gerando algum desconforto que leve ao desmame precoce. Contudo, não há consenso sobre a influência da renda no desmame precoce, pois há estudos que apontam que a renda familiar foi relacionada como fator associado à duração do aleitamento materno total ${ }^{(8)}$. Por outro lado, constatou-se, também por meio de pesquisas, que a renda, assim como a escolaridade materna, não interferiram na duração do aleitamento materno em diferentes grupos sociais $^{(9)}$. Neste estudo, $46,7 \%$ das mães entrevistadas tinham renda familiar menor que um salário mínimo, porém esse dado não teve significância estatística ao ser relacionado com a dor e o desconforto das mesmas.

Embora o parto cesariano tenha sido o mais referido entre as mães, o tipo de parto não teve significância na influência da dor e desconforto que dificultaria a manutenção do aleitamento materno. Outros estudos brasileiros também não encontraram diferença ao comparar a incidência da amamentação e sua manutenção em relação ao tipo de parto ${ }^{(10-11)}$.

Devido à tendência dos neonatos de ficarem mais alertas nas primeiras duas horas de vida, este deve ser considerado um período conveniente para iniciar uma bem-sucedida interação entre a mãe e a criança na amamentação ${ }^{(12)}$. Porém, neste estudo, o local de primeira mamada menos referido pelas mães foi o centro obstétrico, sugerindo que este local deve ser mais estimulado como local de primeira mamada, independentemente do tipo de parto.

A conformação anatômica do mamilo não foi um 
dado estatisticamente significativo ao relacionar com a dor e desconforto, em concordância com a afirmação do Ministério da Saúde ${ }^{(13)}$.

No presente estudo nenhuma mãe referiu adotar uma postura ideal para amamentar. No entanto, a maioria $(95,2 \%)$ relatou ter preferência por amamentar sentada, e destas, o mobiliário escolhido foi cadeira ou poltrona. Considerando que $35,6 \%$ das mães referiram sentir dor ou desconforto em alguma região do corpo, evidencia-se a importância da orientação postural no puerpério. Provavelmente, muitas mães não referiram sentir dor por considerar um processo normal para satisfazer seu filho e família, evidenciando foco maior no vínculo que estão estabelecendo do que propriamente nas dores que estão sentindo ${ }^{(14-16)}$.

Neste estudo houve uma maior prevalência de posição inadequada do bebê, ocasionando queixa de dor e desconforto em 26,1\% das entrevistadas. Quanto à posição do bebê, foi considerada uma posição correta quando este estava com a cabeça alinhada com o corpo, queixo tocando a mama da mãe e cabeça apoiada na região da nuca, por ser importante para uma preensão correta da mama ${ }^{(17)}$.

Ao analisar fatores que poderiam interferir nesse desfecho, para este estudo, $35,7 \%$ das mães entrevistadas referiram dor ou desconforto em alguma região do corpo ao amamentar, visto que a região e a causa mais referida foram as mamas devido a fissuras. Embora tenhamos observado queixa de dor no mamilo, consequência da lesão mamilar, isto não gerou o desmame, porém criou momentos de desconforto para as mães. A amamentação é referida por inúmeras mulheres como um momento sublime e prazeroso, desde que não haja dor $^{(18)}$. A dor nas mamas e a sensação dolorosa nos mamilos são as queixas mais referidas pelas mães e as mais estudadas, pois interferem diretamente na prática do aleitamento materno, visto que estão associadas ao desmame precoce ${ }^{(3,19-20)}$.

$\mathrm{O}$ único fator associado à dor e ao desconforto referidos pelas mães que teve significância estatística foi o sexo da criança $(p=0,038)$. O sexo do bebê foi relacionado com a dor e desconforto, sendo o sexo feminino o mais prevalente nessa relação, sugerindo que o menino pode ter uma melhor sucção ou pega da mama. Não foram encontrados dados na literatura que comparem uma 'boa pega' ou uma sucção mais efetiva entre o sexo masculino e o feminino. Ressaltando, então, a importância da realização de novos estudos para verificar se tais hipóteses fazem sentido.

Outrossim, os profissionais de saúde devem bus- car conhecer as dificuldades das mães no processo de aleitamento materno, para que possam proporcionar alternativas importantes e indispensáveis para evitar o desmame precoce ${ }^{(21)}$. Vale ressaltar que profissionais envolvidos com o aleitamento materno devem trabalhar com criatividade e atualização de conhecimentos diretamente ligados à amamentação, estando atentos e sensíveis às necessidades do binômio mãe-filho ${ }^{(22)}$.

\section{CONCLUSÕES}

De acordo com os dados desta pesquisa, observouse falta de estímulo precoce ao aleitamento materno e ao vínculo mãe-bebê, visto que o centro obstétrico foi o local de primeira mamada menos referido. A idade média das mães foi 23 anos e a falta de experiência com amamentação não foi um fator estatisticamente significativo ao ser relacionado com a dor e o desconforto em alguma região do corpo das lactentes, bem como a conformação anatômica dos mamilos e a posição e pega do lactente.

Nenhuma mãe referiu adotar uma postura ideal para amamentar (sentada, costas recostadas, ombros relaxados, pés apoiados e uso de travesseiro no colo). No entanto, a grande maioria relatou ter preferência por amamentar sentada, e estas indicaram cadeiras ou poltronas como o mobiliário mais utilizado para a prática da amamentação. Do total de mulheres, 35,7\% referiram sentir dor ou desconforto em alguma região do corpo e o local de dor e desconforto mais relatado foi a mama, devido a fissuras. O único fator associado à dor e ao desconforto, referido pelas mães, que teve significância estatística foi o sexo da criança $(p=0,038)$.

Sugerimos um estímulo às ações de educação em saúde durante o pré-natal com orientações não somente sobre amamentação, mas também sobre as técnicas corretas da mesma, para prevenirmos e minimizarmos os desconfortos ligados à mamada, que podem resultar no desmame precoce. Também seria interessante enfatizar a importância do início do aleitamento ainda na sala de parto, independentemente de sua via, visando o fortalecimento precoce do vínculo mãe-bebê.

\section{REFERÊNCIAS}

1. Antunes LS, Antunes LAA, Corvino MPF, Maia LC. Amamentação natural como fonte de prevenção em saúde. Ciênc. saúde colet. 2008;13(1). p. 103-9.

2. Ministério da Saúde (BR). Manual de aleitamento materno. Brasília: UNICEF/Comissão Nacional; 2007a.

Cogitare Enferm. 2014 Jan/Mar; 19(1):136-140 
3. Oliveira JS, Joventino ES, Dodt RG, Veras JEGLF, Ximentes LB. Fatores associados ao desmame precoce entre multíparas. Rev. Rene. 2010;11(4). p. 5-102.

4. Morari-Cassol EG, Campos Júnior D, Haeffner LSB. Desconforto músculo-esquelético no pósparto e amamentação. Online Fisioterapia Brasil. [Internet] 2008;9(1) [acesso em 10 mai 2011]. Disponível: http://biblioteca.unipac.br/cgi-bin/wxis. exe? IsisScript $=$ ph18/003.xis\&cipar $=\mathrm{jf} / \mathrm{phl}$.cip\&boo $\mathrm{l}=\exp \&$ opc $=$ decorado\&exp $=$ DESCONFORTO $\% 20$ MUSCULO-ESQUELETICO\&code $=\&$ lang $=$

5. Ministério da Saúde (BR). Ações de promoção de incentivo ao aleitamento. Brasília; 2006 [Internet] [acesso em 10 dez 2009]. Disponível: http://portal.saude.gov.br/ portal/saude/cidadao/visualizar_texto.cfm?idtxt $=24215$.

6. Moreira MA. Os novos significados da amamentação em primíparas que vivenciaram fissuras mamárias, na perspectiva de gênero. In: Fazendo Gênero - Corpo, Violência e Poder, 8. Florianópolis, 2008. Anais. Florianópolis: UFSC [Internet] 2008 [acesso em $15 \mathrm{abr}$ 2011]. Disponível: http://www.fazendogenero.ufsc.br/8/ sts/ST58/Michelle_Araujo_Moreira_58.pdf.

7. Baptista GH, Andrade AHHK, Gonçalves SR. Fatores associados à duração do aleitamento materno em crianças de famílias de baixa renda da região sul da cidade de Curitiba, Paraná, Brasil. Cad. Saúde Pública. 2009;25(3):596-604.

8. Rolla TS, Gonçalves VM da S. Aleitamento materno e seus determinantes. Rev. Enferm. Integrada. [Internet] 2012;5(1) [acesso em $10 \mathrm{dez}$ 2012]. Disponível: http:// www.unilestemg.br/enfermagemintegrada/artigo/ v5/03-aleitamento-materno-e-seus-determinantes.pdf.

9. Fujimori E, Minagawa ÁT, Laurenti D, Montero RMJM, Borges ALV, Oliveira IMV. Duração do aleitamento materno em menores de dois anos de idade em Itupeva, São Paulo, Brasil: há diferenças entre os grupos sociais? Rev. Bras. Saude Mater. Infant. 2010;10(1) [acesso em 15 ago 2012]. Disponível: http://dx.doi.org/10.1590/ S1519-38292010000100004

10. Venancio SI, Escuder MM, Saldiva SRDM, Giugliani ERJ. A prática do aleitamento materno nas capitais brasileiras e distrito federal: situação atual e avanços. J. Pediatr. 2010;86(4).

11. Lima CM, Lira CIP, Vasconcelos LGM. Duração e fatores associados ao aleitamento materno em crianças menores de 24 meses de idade no estado de Pernambuco. Rev. Bras. Saude Mater. Infant. 2006;6(1).
12. Anderson GC, Moore ER, Bergman N. Early skin to skin contact for mothers and their healthy newborn infants. (Cochrane Review). In: Cochrane Database of Systematic Reviews, Issue 3. Oxford: Update Software; 2007.

13. Ministério da Saúde (BR). Secretaria de Atenção à Saúde. Promovendo o aleitamento materno. $2^{\mathrm{a}}$ ed. rev. Brasília: Ministério da Saúde; 2007b.

14. Müller FS, Silva IA. Representações sociais de um grupo de mulheres/ nutrizes sobre o apoio à amamentação. Rev. Latino-Am. Enfermagem. 2009;17(5). p. 651-7.

15. Shimoda GT, Silva IA. Necessidades de saúde de mulheres em processo de amamentação. Rev. bras enferm. [Internet] 2010;63(1) [acesso em 16 nov 2010]. Disponível: http://dx.doi.org/10.1590/S003471672010000100010

16. Piccinini CA, Lopes RS, Grill AG, De Nardi T. Gestação e constituição da maternidade. Psicologia em Estudo. 2008;13(1).

17. Nóbrega TP. Corpo, percepção e conhecimento em Merleau-Ponty. Estud. Psicol. 2008;13(2). p. 141-8.

18. Gonçalves AS, Filipini R, Posso MBS. Dor mamilar durante a amamentação: ação analgésica do laser de baixa intensidade. Rev. dor. 2009;2:125-9.

19. Machado MMT, Bossi MLM. Compreendendo a prática do aleitamento exclusivo: um estudo junto a lactantes usuárias da Rede de Serviços em Fortaleza, Ceará, Brasil. Rev. Bras. Saude Mater. Infant. 2008;8(2). p.187-96.

20. Montrone AVG, Arantes CIS, Nassar ACS, Zanon T. Trauma mamilar e a prática de amamentar: estudo com mulheres no início da Lactação. Rev. APS. 2006;9(2):168-74.

21. Frota MA, Aderaldo NNS, Silveira VG, Rolim KMC, Martins MC. O reflexo da orientação na prática do aleitamento materno. Cogitare enferm. 2008;13(3):403-9.

22. Pasqual KK, Braccialli LAD, Volponi M. Alojamento conjunto: espaço concreto de possibilidades e o papel da equipe multiprofissional. Cogitare enferm. 2010;15(2):334-9. 